\title{
Identifying Symmetry and Contracted Ratio of Lateral Abdominal Muscles in Stroke Survivors
}

\author{
Dongkwon Seo ${ }^{1}$, Seungwon Lee ${ }^{2, *}$ and Yeonseop Lee ${ }^{3}$ \\ ${ }^{1}$ Department of Physical Therapy, Konyang University, Daejeon, Korea \\ ${ }^{2}$ Department of Physical Therapy, Sahmyook University, Seoul, Korea \\ ${ }^{3}$ Department of Physical Therapy, Daewon University, Chungbuk, Korea \\ "Corresponding author's E-mail: swlee@syu.ac.kr
}

\begin{abstract}
The purpose of this study was to measure the lateral abdominal muscles (LAM) in stroke patients and the healthy adults and compare the symmetry and contracted ratio of LAM thickness of two groups. Twenty-six stroke patients and 35 healthy adults were participated. The thicknesses of transverse abdominis (TrA), internal oblique (IO), and external oblique (EO) muscles on the paretic/non-paretic (left/right) sides were measured by using ultrasonography during contraction and rest period. After measurements, we calculated symmetry and contracted ratio of LAM. Comparison of symmetric ratio of the two groups showed significant difference in all abdominal muscles, but not EO during contraction. Comparison of contracted ratio of the two groups showed significant difference in TrA, but not IO and EO. This study was investigated an asymmetry of LAM and decline of contracted ratio of TrA in stroke patients. These results revealed that recovery of $\operatorname{Tr} A$ is needed for stroke patients. Further study on specific exercise for recovery of TrA in stroke patients will have to be conducted.
\end{abstract}

Keywords: stroke, lateral abdominal muscles, asymmetry, ultrasonography

\section{Introduction}

Stroke patients have various functional problems due to the asymmetric paretic and non-paretic side. Stroke patients have decreased voluntary ventilation on the paretic side, compared to the non-paretic side, and normal respiration does not occur due to asymmetric ventilation [1]. Decreased cognition and knee extensor strength asymmetry in stroke patients cause weight-bearing asymmetry in activities of daily living, such as sit-to stand, compared to normal healthy people [2]. Stroke patients also experience problems in functional activity due to weight asymmetry, where $60 \sim 90 \%$ of the weight is shifted to the non-paretic side during standing or gait [3]. This asymmetry of weight-bearing is deeply related to trunk asymmetry. Stroke patients have trunk asymmetry, inability to maintain the midline due to injured trunk position sense in the transverse and sagittal plane, damaging trunk performance, and negatively affecting balance and gait $[4,5]$. In addition, trunk performance and control play a leading part in predicting function of stroke patients in activities of daily living (ADL), and early management of trunk control is important for rehabilitation of stroke patients [6], [7]. Therefore, trunk control, trunk performance, and trunk symmetry play an important role in functional recovery of stroke patients.

Trunk muscles provide stability as a prime mover, by contracting first when the body or extremities move or as an anticipatory postural adjuster while the extremities move, and function in proper performance of the trunk [8]. However, stroke patients show delayed contraction in the affected the latissimus dorsi (LD), external oblique (EO), and rectus abdominis muscles (RA), showing decreased function as a trunk stabilizer, affecting functional activities and showing compensatory patterns $[9,10]$. In addition, EO of the 
non-paretic side was compensated for RA deficit in stroke patients during therapeutic exercise. Therefore, selection of the proper exercise method is important [11].

Superficial muscles such as RA and EO of trunk muscles are measured by surface electromyography (sEMG) study in order to understand the problems of the muscles, therefore, diagnosis and research are possible [9, 10]. Transversus abdominal muscle (TrA), the prime mover that provides stability to the spine, is a deep muscle, therefore, invasive methods, such as needle EMG, provide more accurate measurements. However, pain and discomfort are a problem [8]. With other methods, diagnosis can be made with computed tomography (CT), however, there is a risk of danger of radiation exposure [12].

To overcome this problem, ultrasound images can be used for measurement of core muscles. Various methods have been used recently. In the beginning, the ultrasound image of trunk muscles was used as an assistive method for accurate placement of the needle of needle EMG in the deep TrA muscles [8]. However, ultrasound images are used specifically for training and learning movements by visual feedback during therapeutic exercise, not just for studies on muscle thickness or symmetry using images of the internal oblique (IO), EO, and TrA, which provide trunk stability through functional coordination activities; it is also commonly used before and after treatment for evaluation of treatment effects, and is used as a training tool [13-15]. Changes in TrA and multifidus muscle (MS) were observed on ultrasound images of patients with lower back pain, compared to normal people, and it was an important factor in these findings [16, 17]. Ultrasonography is easily used noninvasively, has no risk of radiation, and is more economical than CT; therefore, used well. However, few studies have reported on changes in trunk muscles of stroke patients using ultrasound images, and there is no aging-matched study. Therefore in this study, stroke patients and aging-matched people's trunk muscles (TrA, IO, and EO) were measured through ultrasound images. Thickness, symmetry ratio, and contracted ratio were calculated and compared between the both groups.

\section{Methods}

\subsection{Participants}

Twenty six stroke patients treated at $\mathrm{K}$ University Hospital and 35 aging matched adults who live in the community participated in this study. All subjects provided written informed consent prior to participation. The inclusion criteria of the stroke group (SG) were subjects who agreed to participate, sick-leave due to stroke for more than one and one half years at presentation, were able to walk without assistance, had no orthopedic problems that affected the trunk, had no chronic lower back pain, and who understood the directions (MMSE-K > 24 point), with no unilateral neglect, hemianopsia, or apraxia, and no psychological or emotion problems. The inclusion criteria of the healthy adult group (AG) subjects who agreed to participate, were able to walk without assistance, had no orthopedic problems that affected the trunk, had no chronic lower back pain, and who understood the directions. The study protocol was approved by the institutional review board.

\subsection{Ultrasonography}

Ultrasonography (MySono U5, Samsung Medison Co., Seoul, Korea) equipment was used in this study. Using a 7.5 MHz linear transducer, the thicknesses of TrA, IO, and EO on the paretic and the non-paretic sides in SG and on the left and right sides in AG were measured during the contraction and rest periods. Abdominal draw-in maneuver (ADIM) was performed for measurement of contraction position. The transducer head was placed $25 \mathrm{~mm}$ internally between the 12th rib and iliac crest, and measured twice; contraction and rest period (Figure 1). 


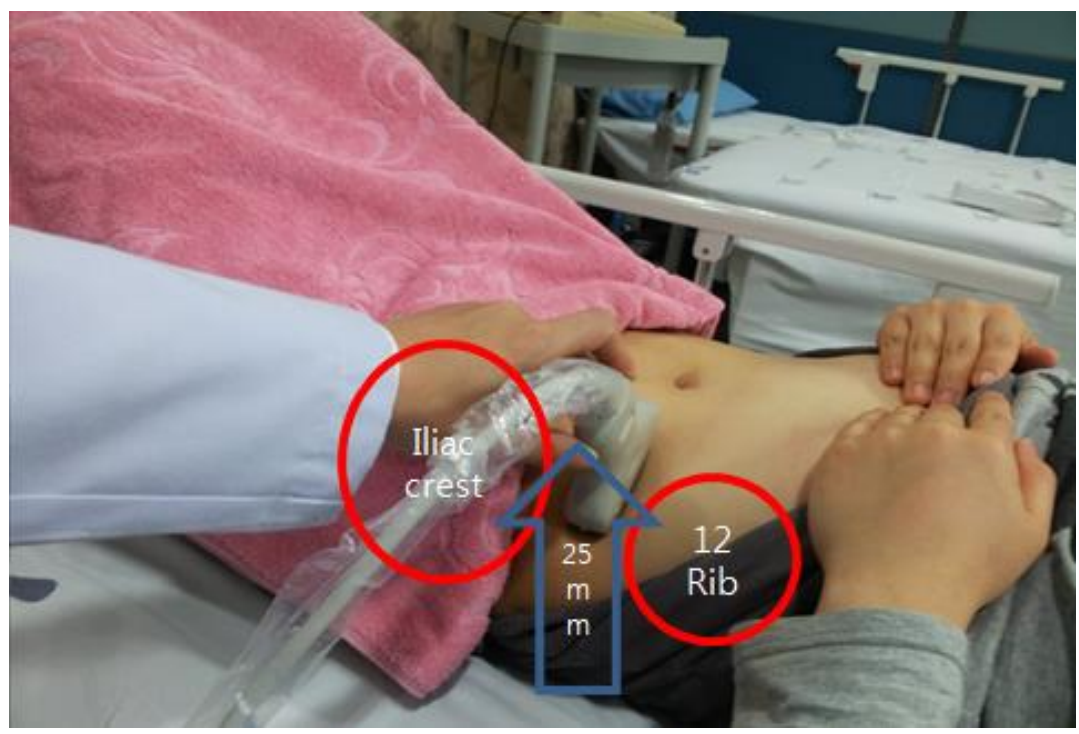

Figure 1. The Transducer Placement to Measure Lateral Abdominal Muscles

Each measurement was repeated three times, and the average was used. For contraction, subjects lie supine, comfortably flexing the hip joint and knee joint, minimizing lordosis, and pull up the lower abdomen $[14,18]$. Five minutes were given before the measurement in order to allow practice for accurate posture. For contraction (ADIM) practice, patients looked at the monitor screen, and the therapist provided visual feedback for accurate contraction by looking into the monitor. After ultrasound imaging measurement, muscle thickness was measured by drawing a line from the left end to the right end of the image, $1.5 \mathrm{~cm}$ apart from the muscle-fascia junction, and a horizontal line was drawn for measurement of TrA, IO, and EO, which were measured in order [19](Figure 2).

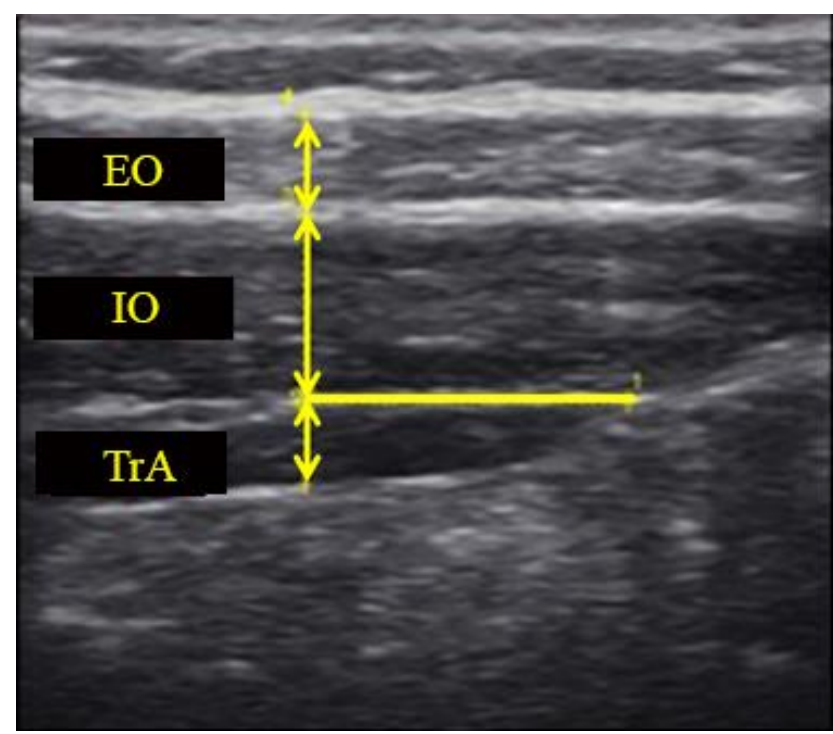

Figure 2. Measurement of thickness of lateral abdominal muscles $\mathrm{EO}=$ external oblique muscle, $\mathrm{IO}=$ internal oblique muscle, $\operatorname{Tr} \mathrm{A}=$ transverse abdominis 


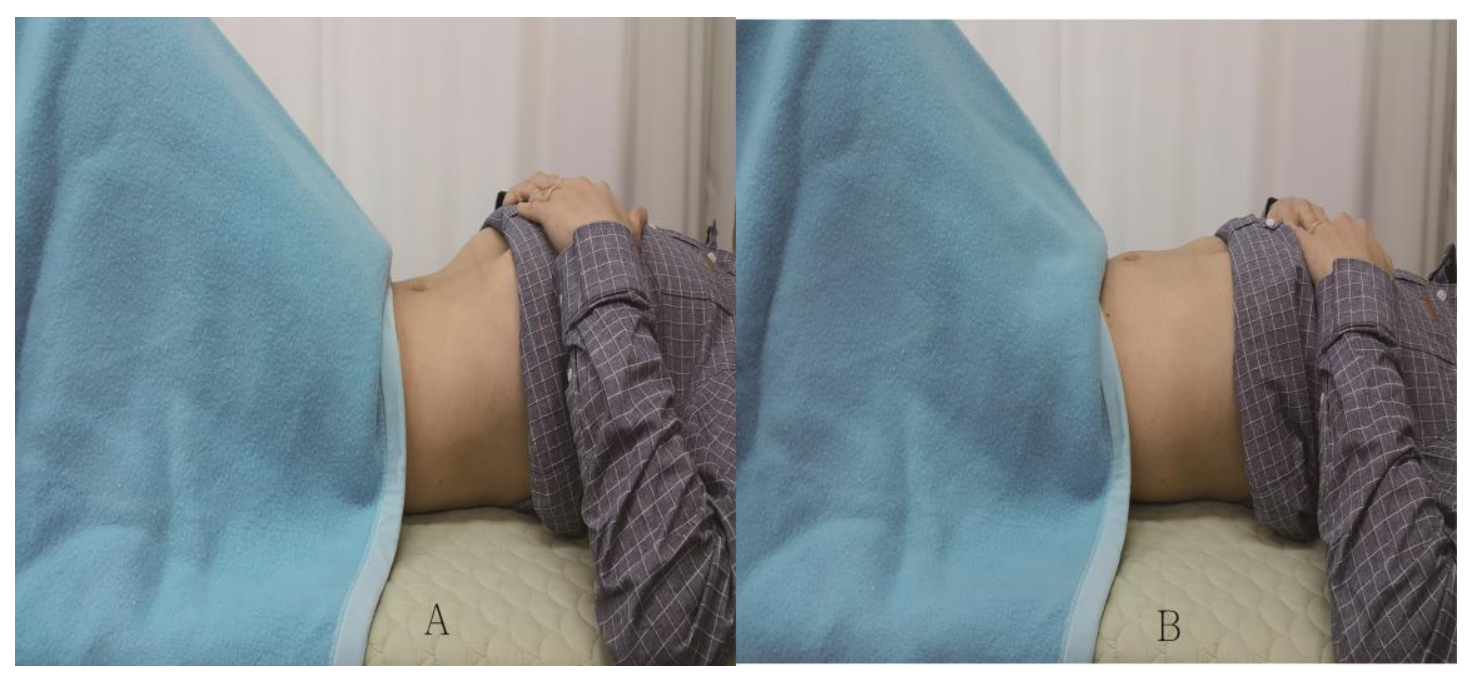

Figure 3. Patient's Positions to Measure Lateral Abdominal Muscles $A=A D I M$ (contraction) position, $B=$ resting position .

\subsection{Experimental Methods}

Subjects were positioned in a crook-lying supine position with their hip and knee flexed. Their arms were positioned straight on the bed. The lumbar spine was in neutral position. During this study, two postures were assessed; (1) rest, (2) contraction. Rest is measured in expiration of a normal respiratory pattern, and contraction is measured on maintaining respiration after abdominal draw - in maneuver (ADIM) on 2/3 of normal expiration. Rest expiration position was used as a baseline measure for calculating the change in $\operatorname{TrA}$, IO, and EO. All positions were repeated three times and performed randomly. Subjects practiced each position prior to data procedure. The verbal instruction for ADIM was "hold breathe after tidal expiration, and then move your navel toward the lower back". ADIM was performed without movement of the lumbar spine, pelvis, and rib cage. This study compared symmetric and contracted ratio after measurement of thickness of LAM. Symmetric ratio is calculated by ratio (dominant / non-dominant) (non-paretic side / paretic side), contracted ratio is calculated by ratio (contraction / rest) [14, 18](Figure 3).

\subsection{Statistical Analysis}

In this study, SPSS 15.0 for Windows was applied for statistical analysis of the results. Data normality was tested with Shapiro - Wilk; all variables were normal distribution. Independent $\mathrm{t}$ - test and Chi test were used for the homogeneity test. An independent $\mathrm{t}$ test was performed for comparison of the thicknesses lateral abdominal muscles of each group. An independent $\mathrm{t}$ - test was performed for comparison of symmetric and contracted ratio of the lateral abdominal muscles of the two groups. The alpha level was set at 0.05 for all analyses.

\section{Results}

\subsection{General Information}

In comparison of the general characteristics of SG and AG individuals, age, weight, and height were not significantly different. Characteristics of SG were shown in the Table 1. 
Table 1. General Characteristics of the Subjects $(\mathrm{N}=61)$

\begin{tabular}{|c|c|c|c|c|}
\hline & $S G(n=26)$ & $A G(n=35)$ & $x^{2 / t}$ & $\mathrm{p}$ \\
\hline Gender (Male/Female) & $19 / 7$ & $12 / 23$ & 3.643 & .07 \\
\hline Age (years) & $57.96 \pm 13.49$ & $61.94 \pm 6.82$ & 2.226 & .14 \\
\hline Weight (kg) & $62.96 \pm 8.31$ & $60.69 \pm 7.14$ & 1.310 & .25 \\
\hline Height $(\mathrm{cm})$ & $163.04 \pm 7.34$ & $159.69 \pm 8.63$ & 2.485 & .11 \\
\hline $\begin{array}{l}\text { Side of hemiplegia } \\
\text { (left/right) }\end{array}$ & $14 / 12$ & - & - & - \\
\hline $\begin{array}{l}\text { Lesion type } \\
\text { (infarction/hemorrhage) }\end{array}$ & $14 / 12$ & - & - & - \\
\hline $\begin{array}{l}\text { Onset duration } \\
\text { (months) }\end{array}$ & $20.31 \pm 33.52$ & - & - & - \\
\hline MMSE-K (score) & $27.50 \pm 1.84$ & - & - & - \\
\hline
\end{tabular}

Note: Values indicate mean \pm standard deviation. $S G=$ stroke group, $A G=$ adult group.

\subsection{Comparison of the Symmetry of Abdominal Muscles}

Symmetry of the two groups (non-paretic side / paretic side or dominant / nondominant), was compared, and showed significant difference in TrA (rest), IO (rest), EO (rest), TrA (ADIM), and IO (ADIM) $(\mathrm{p}<0.05)$, but not EO (ADIM) $(\mathrm{p}>0.05)$. Therefore, stroke patients showed larger differences than elderly individuals (Table 2).

\subsection{Comparison of the Contracted Ratio of Abdominal Muscles}

In comparison between the two, the contracted ratio (contraction/rest) showed significant difference between the Paretic $\operatorname{Tr} A$ and Non-paretic $\operatorname{Tr} A(p<0.05)$, but not IO and EO ( $p>0.05)($ Table3).

Table 2. Comparison of the Symmetry of Abdominal Muscles $(\mathrm{N}=61)$

\begin{tabular}{lcccc}
\hline & & $\mathrm{SG}(\mathrm{n}=26)$ & $\mathrm{AG}(\mathrm{n}=35)$ & $\mathrm{p}$ \\
\hline Rest & & & & \\
& TrA & $1.21 \pm 0.15$ & $0.98 \pm 0.17$ & $5.384^{*}(.00)$ \\
& IO & $1.19 \pm 0.15$ & $1.04 \pm 0.20$ & $3.108^{*}(.00)$ \\
& EO & $1.11 \pm 0.11$ & $0.99 \pm 0.28$ & $2.376^{*}(.03)$ \\
$\mathrm{ADIM}$ & & & & \\
& TrA & $1.13 \pm 0.13$ & $1.00 \pm 0.21$ & $2.810^{*}(.00)$ \\
& IO & $1.11 \pm 0.11$ & $0.97 \pm 0.15$ & $3.740^{*}(.01)$ \\
& EO & $1.09 \pm 0.11$ & $1.06 \pm 0.29$ & $.591(.55)$ \\
\hline
\end{tabular}


Note. Values are expressed as mean \pm standard deviation. $S G=$ stroke group, $A G=$ adult group, $\operatorname{Tr} \mathrm{A}=$ transverse abdominal muscle, $\mathrm{IO}=$ internal oblique muscle, $\mathrm{EO}=$ external oblique muscle, $A D I M=$ abdominal draw-in maneuver. ${ }^{*}$ Significant differences between $S G$ and $A G(p<.05)$.

Table 3. Comparison of the Contracted Ratio of Abdominal Muscles ( $N=61)$

\begin{tabular}{|c|c|c|c|}
\hline & $S G(n=26)$ & $A G(n=35)$ & $p$ \\
\hline \multicolumn{4}{|l|}{ TrA } \\
\hline Paretic & $1.42 \pm 0.19$ & $1.60 \pm 0.41$ & $2.028^{*}(.04)$ \\
\hline Non-paretic & $1.45 \pm 0.22$ & $1.69 \pm 0.36$ & $3.285^{*}(.00)$ \\
\hline \multicolumn{4}{|l|}{10} \\
\hline Paretic & $1.30 \pm 0.22$ & $1.30 \pm 0.46$ & $1.081(.28)$ \\
\hline Non-paretic & $1.31 \pm 0.17$ & $1.41 \pm 0.31$ & $1.042(.16)$ \\
\hline \multicolumn{4}{|l|}{ EO } \\
\hline Paretic & $1.08 \pm 0.13$ & $1.05 \pm 0.15$ & $-0.616(.54)$ \\
\hline Non-paretic & $1.06 \pm 0.11$ & $1.15 \pm 0.34$ & 1.059 (.29) \\
\hline
\end{tabular}

Note. Values are expressed as mean \pm standard deviation. $S G=$ stroke group, $A G=$ adult group, $\operatorname{Tr} A=$ transverse abdominal muscle, $I O=$ internal oblique muscle, $E O=$ external oblique muscle. *Significant differences between $S G$ and $A G(p<.05)$.

\section{Discussion}

This study was conducted in order to determine the trunk muscle asymmetry affecting the balance, gait, and ADL of stroke patients. Ultrasound imaging was used for measurement of TrA, IO, and EO muscles. Calculation of symmetry and contracted ratio through thickness of LAM and comparison between the both groups were then performed. However, most trunk muscle - related studies used surface EMG for analysis of the effects. Surface EMG on the trunk muscles is problematic due to interruption caused by overflow of data from other muscles. Therefore, it was not sufficient for acquisition of accurate data, which presents the problem of using a needle for measurement of $\operatorname{Tr} A$, the prime mover. Therefore, this study measured trunk muscles using ultrasound imaging in order to overcome the weakness of EMG. EMG showed non - significant activation between stroke patients and normal subjects. However, the reason for choosing the abdominal muscles instead of lumbar erecter spinae is that abdominal muscles act as an anticipatory postural adjuster, providing stability of the trunk. For this reason, TrA, IO, and EO were selected in the study $[9,10]$. Intraclass correlation coefficients are excellent in measurement of trunk muscle using ultrasound images [20].

In this study, as a method for analysis of measured thickness, symmetry is used because use of personal ratio cannot compare the factors between subjects. Contracted ratio is used because muscle thicknesses cannot be compared, and each ratio was calculated, normalized, and then compared [14, 18, 20].

The study result showed a significantly higher ratio. Among the contracted ratio of SG, TrA ratio was approximately 1.4 , AG showed 1.6 times the value, showing the highest ratio. This result supports those from previous studies showing that stroke patients have delayed onset time, lower ratio on EMG, compared to normal subjects $[9,10,21,22]$, and trunk strength of stroke patients was decreased, compared with normal elderly people [23]. 
In a study of 10 acute, 10 subacute, and 10 chronic phase stroke patients for measurement of TrA, IO, and EO, Seo (2013) found that the longer the stroke period, acute, subacute, and chronic phase, contracted ratio of $\operatorname{TrA}$ on the paretic side was lower than that on the non - paretic side [24].

A previous study analyzed the contracted ratio, and reported that $\operatorname{Tr} A$ ratio for subjects with chronic lower back pain was approximately 1.65-1.75, similar to that of the elders, however, a difference was observed in stroke patients [20]; in a study with other normal subjects, approximately 1.4 was obtained, and values similar to those of stroke patients were recorded $[13,14]$. However, in the studies mentioned above, only a wide range of subjects, from teenagers to those in their late $60 \mathrm{~s}$, were studied; direct comparison of the results of aging matched in this study is difficult, however the comparison meaningful. It will help clinicians in making decisions.

The results of this study, particularly those regarding symmetry, showed that stroke patients had more asymmetric TrA, IO, and EO, compared to healthy adults. Non - paretic TrA ratio had a larger contraction rate, and TrA ratio of the paretic side had a smaller contraction ratio.

Results like these demonstrated that stroke patients had more asymmetric TrA, compared to the aged, and asymmetric contraction was observed. In previous studies, stroke patients had weaker muscle power than normal subjects; the weakness was in the paretic side, compared to the non-paretic side, and delayed contraction was observed [23, 25]. These stroke patients have decreased trunk control, cannot perform as a prime mover, and these were the results. The results will provide basic data for therapeutic exercise for recovery of TrA in stroke patients.

Regarding the limitations of this study, first, the correlation of asymmetric trunk and body weight (body mass index) should not be determined and compared. Second, study on the effects of decreased asymmetry ratio in the trunk muscles, and functional abilities (balance, gait) and analysis of their correlations should be performed in order to determine how they affect decreased ratio; however, this work was not completed. Finally, correlation between ultrasound imaging and EMG should be determined. Further studies should be conducted in order to overcome these limitations.

\section{Conclusions}

This study found that asymmetry in LAM, and contracted ratio of TrA was decreased in stroke patients. These results indicate that recovery of TrA is needed for stroke patients. Further study will be conducted for specific exercise for recovery of TrA in stroke patients.

\section{References}

[1] B. Lanini, R. Bianchi, I. Romagnoli, C. Coli, B. Binazzi, F. Gigliotti, A. Pizzi, A. Grippo and G. Scano, "Chest wall kinematics in patients with hemiplegia", Am. J. Respir. Crit. Care Med., vol. 168, (2003), pp. 109-113.

[2] A. Briere, S. Lauziere, D. Gravel and S. Nadeau, "Perception of weight-bearing distribution during sitto-stand tasks in hemiparetic and healthy individuals", Stroke, vol. 41, (2010), pp. 1704-1708.

[3] Y. Laufer, R. Dickstein, S. Resnik and E. Marcovitz, "Weight-bearing shifts of hemiparetic and healthy adults upon stepping on stairs of various heights", Clin. Rehabil., vol. 14, (2000), pp. 125-129.

[4] S. Ryerson, N. N. Byl, D. A. Brown, R. A. Wong and J. M. Hidler, "Altered trunk position sense and its relation to balance functions in people post-stroke", J. Neurol. Phys. Ther., vol. 32, (2008), pp. 14-20.

[5] G. Verheyden, L. Vereeck, S. Truijen, M. Troch, I. Herregodts, C. Lafosse, A. Nieuwboer and W. De Weerdt, "Trunk performance after stroke and the relationship with balance, gait and functional ability", Clin. Rehabil., vol. 20, (2006), pp. 451-458.

[6] C. L. Hsieh, C. F. Sheu, I. P. Hsueh and C. H. Wang, "Trunk control as an early predictor of comprehensive activities of daily living function in stroke patients", Stroke, vol. 33, (2002), pp. 26262630. 
[7] E. Duarte, E. Marco, J. M. Muniesa, R. Belmonte, P. Diaz, M. Tejero and F. Escalada, "Trunk control test as a functional predictor in stroke patients", J. Rehabil. Med., vol. 34, (2002), pp. 267-272.

[8] P. W. Hodges and C. A. Richardson, "Contraction of the abdominal muscles associated with movement of the lower limb", Phys. Ther., vol. 77, (1997), pp. 132-142.

[9] R. Dickstein, S. Shefi, E. Marcovitz and Y. Villa, "Anticipatory postural adjustment in selected trunk muscles in post stroke hemiparetic patients", Arch. Phys. Med. Rehabil., vol. 85, (2004), pp. 261-267.

[10] R. Dickstein, S. Shefi, E. Marcovitz and Y. Villa, "Electromyographic activity of voluntarily activated trunk flexor and extensor muscles in post-stroke hemiparetic subjects", Clin. Neurophysiol., vol. 115, (2004), pp. 790-796.

[11] L. M. Pereira, F. C. Marcucci, M. de Oliveira Menacho, M. R. Garanhani, E. L. Lavado and J. R. Cardoso, "Electromyographic activity of selected trunk muscles in subjects with and without hemiparesis during therapeutic exercise", J. Electromyogr. Kinesiol., vol. 21, (2011), pp. 327-332.

[12] T. Tsuji, M. Liu, K. Hase, Y. Masakado and N. Chino, "Trunk muscles in persons with hemiparetic stroke evaluated with computed tomography", J. Rehabil. Med., vol. 35, (2003), pp. 184-188.

[13] A. F. Mannion, N. Pulkovski, V. Toma and H. Sprott, "Abdominal muscle size and symmetry at rest and during abdominal hollowing exercises in healthy control subjects", J. Anat., vol. 213, (2008), pp. 173182.

[14] A. F. Mannion, N. Pulkovski, D. Gubler, M. Gorelick, D. O'Riordan, T. Loupas, P. Schenk, H. Gerber and H. Sprott, "Muscle thickness changes during abdominal hollowing: an assessment of between-day measurement error in controls and patients with chronic low back pain", Eur. Spine J., vol. 17, (2008), pp. 494-501

[15] S. M. Henry and K. C. Westervelt, "The use of real-time ultrasound feedback in teaching abdominal hollowing exercises to healthy subjects", J. Orthop. Sports Phys. Ther., vol. 35, (2005), pp. 338-345.

[16] P. W. Hodges and C. A. Richardson, "Delayed postural contraction of transversus abdominis in low back pain associated with movement of the lower limb", J. Spinal Disord., vol. 11, (1998), pp. 46-56.

[17] P. W. Hodges and C. A. Richardson, "Inefficient muscular stabilization of the lumbar spine associated with low back pain", A motor control evaluation of transversus abdominis, Spine (Phila Pa 1976), vol. 21, (1996), pp. 2640-2650.

[18] D. M. Stetts, J. E. Freund, S. C. Allison and G. Carpenter, "A rehabilitative ultrasound imaging investigation of lateral abdominal muscle thickness in healthy aging adults", J. Geriatr. Phys. Ther., vol. 32, (2009), pp. 60-66.

[19] P. W. Hodges, L. H. Pengel, R. D. Herbert and S. C. Gandevia, "Measurement of muscle contraction with ultrasound imaging, Muscle Nerve, vol. 27, (2003), pp. 682-692.

[20] S. L. Koppenhaver, J. J. Hebert, J. M. Fritz, E. C. Parent, D. S. Teyhen and J. S. Magel, "Reliability of rehabilitative ultrasound imaging of the transversus abdominis and lumbar multifidus muscles", Arch. Phys. Med. Rehabil., vol. 90, (2009), pp. 87-94.

[21] R. Dickstein, S. Sheffi, Z. Ben Haim, E. Shabtai and E. Markovici, "Activation of flexor and extensor trunk muscles in hemiparesis", Am. J. Phys. Med. Rehabil., vol. 79, (2000), pp. 228-234.

[22] R. Dickstein, Y. Heffes, Y. Laufer and Z. B. Haim, "Activation of selected trunk muscles during symmetric functional activities in poststroke hemiparetic and hemiplegic patients", J. Neurol. Neurosurg Psychiatry, vol. 66, (1999), pp. 218-221.

[23] M. Karatas, N. Cetin, M. Bayramoglu and A. Dilek, "Trunk muscle strength in relation to balance and functional disability in unihemispheric stroke patients", Am. J. Phys. Med. Rehabil., vol. 83, (2004), pp. 81-87.

[24] D. Seo, S. Lee and O. Kwon, "Comparison of the changes in thickness of the abdominal wall muscles of stroke patients according to the duration of their illness as observed using ultrasonographic images", J Phys Ther Sci, vol. 25, (2013), pp. 817-819.

[25] S. J. Cho, "Current Research Trend of Bioscience and Welfare II", Proceedings of the International Workshop Bioscience and Medical Research, (2015); Jeju, Korea. 